\title{
Breakdown of adiabatic invariance in spherical tokamaks
}

\author{
Johan Carlsson \\ Oak Ridge National Laboratory, P.O. Box 2009, Oak Ridge, TN 37831-8071, USA
}

August 19, 2021

\begin{abstract}
Thermal ions in spherical tokamaks have two adiabatic invariants: the magnetic moment and the longitudinal invariant. For hot ions, variations in magnetic-field strength over a gyro period can become sufficiently large to cause breakdown of the adiabatic invariance. The magnetic moment is more sensitive to perturbations than the longitudinal invariant and there exists an intermediate regime, super-adiabaticity, where the longitudinal invariant remains adiabatic, but the magnetic moment does not. The motion of super-adiabatic ions remains integrable and confinement is thus preserved. However, above a threshold energy, the longitudinal invariant becomes non-adiabatic too, and confinement is lost as the motion becomes chaotic. We predict beam ions in present-day spherical tokamaks to be super-adiabatic but fusion alphas in proposed burning-plasma spherical tokamaks to be non-adiabatic.
\end{abstract}

Consider a charged particle, with mass $m$ and charge $q$, gyrating with speed $v$ in a magnetic field, with field strength $B$. Assume axi-symmetric toroidal geometry with coordinates $(R, \phi, Z)$. The motion preserves the energy $E$ (because the Lorentz force performs no work) and canonical toroidal momentum $P_{\phi}$ (because the toroidal coordinate is ignorable). There is no third exact constant of motion, but the normalized magnetic moment

$$
\Lambda=\frac{B_{0} \mu}{E}=\frac{B_{0} v_{\perp}^{2}}{B v^{2}}
$$

where $v_{\perp}$ is the velocity component perpendicular to the magnetic field $\left(v_{\perp}^{2}=v^{2}-v_{\|}^{2}, v_{\|}=\mathbf{v} \cdot \mathbf{B} / B\right)$ and $B_{0}$ is the on-axis magnetic field, is an adiabatic invariant, i.e. as long as the variation in the magnetic field experienced by the particle during one gyro period is small, $\Lambda$ oscillates at the gyro frequency around a constant average. If we introduce the adiabaticity parameter, $\varepsilon$, the condition of slow (adiabatic) variation becomes

$$
\varepsilon=\frac{\varrho|\nabla B|}{B} \ll 1,
$$

where the gyro radius $\varrho=v_{\perp} / \Omega$, with the gyro frequency $\Omega=|q| B / m$. The approximate constancy of the magnetic moment was first pointed out by Alfvén [1]. Just as the magnetic moment is associated with the gyro motion, a second adiabatic invariant is associated with the slower drift motion: the longitudinal invariant $J_{\|}=\oint v_{\|} d \ell$ [2].

When $\varepsilon \ll 1$, there are thus three constants of motion, one for each degree of freedom, i.e. the motion is integrable, the orbits are quasi-periodic and, in the absence of collisions, a particle will be eternally confined. As the perturbation grows and $\varepsilon \lesssim 1$, a dramatic change in the character of the motion, from bounded to unbounded, will occur, as the adiabaticity breaks down, the third constant of motion is lost, the motion becomes non-integrable, and the orbits become chaotic. This process was first investigated numerically [3] and later fully explained by the rigorous KAM (Kolmogorov, Arnold \& Moser) theory [4]. Subsequently, Chirikov provided an approximate, but more practical, approach when he introduced his semi-empirical resonance-overlap criterion and the standard mapping [5]. In the Chirikovian model, the breakdown of adiabaticity is caused by strong nonlinear resonance between the periodic motions occurring on different timescales. 
The compact and low-field spherical tokamak was introduced by Peng and Strickler [6]. The first experiment, START [7], showed encouraging results and has been succeeded by two larger machines, MAST [8] and NSTX [9]. Larger, burning-plasma, experiments have also been proposed [10]. The $B$ contours of spherical tokamaks are characterized by a magnetic well, i.e. a local minimum in $B$, centered outside of the magnetic axis [see Fig. 1]. For the case shown here, $B_{\min }=0.21 \mathrm{~T}$ and $B_{\max }=1.26 \mathrm{~T}$. The low value and large variation of $B$ in the plasma (a factor of six) leads to a low threshold energy for the onset of non-adiabatic motion. Furthermore, the threshold energy will vary substantially over the cross section. We will demonstrate the consequences of this by solving the Lorentz equation of motion for $80 \mathrm{kV}$ deuterium ions moving in the NSTX magnetic equilibrium shown in Fig. 1.

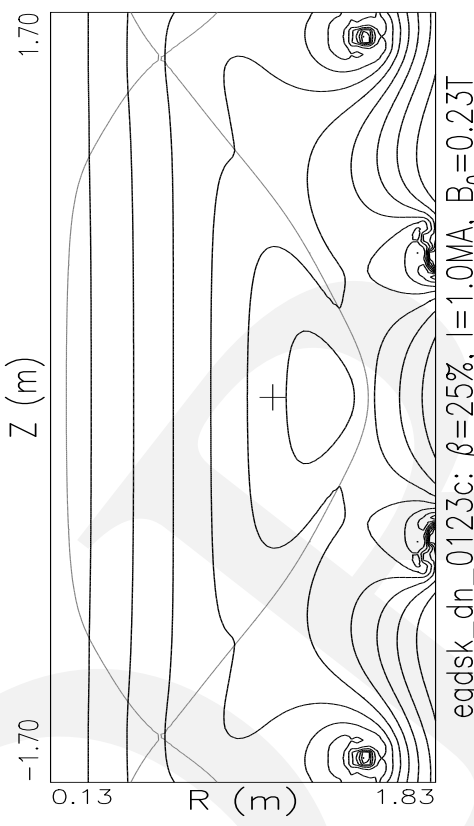

Figure 1: 25\% $\beta$ NSTX equilibrium. Adjacent contours are equidistant in $1 / B$. The thin, gray line is the separatrix and the cross marks the magnetic axis. The poloidal-field coils, carrying approximately 200 $\mathrm{kA}$ of current each, are easily identifiable to the right.
At NSTX, co-injection of an $80 \mathrm{kV}$ deuterium beam is used to heat the plasma (co-injection is favored over counter-injection because it results in lower prompt losses) [9]. Note that the beam directions are relative to the plasma current, which is in the opposite direction of the toroidal magnetic field, and a co-injected ion thus has $v_{\|}<0$. Here, however, we will first launch a counter-injected ion, born with its gyro center just below the midplane at $R_{g c}=0.70 \mathrm{~m}$. The resulting orbit is shown in Fig. 2. Plotting the time evolution of the normalized magnetic moment $\Lambda$ [see Fig. 3], the adiabaticity is evident; $\Lambda$ oscillates around the constant mean with the amplitude spiking when the ion passes through the minimum of $B$ along the orbit. The longitudinal invariant $J_{\|}$is practically constant.

Next, we launch a co-injected ion with its gyro center at exactly the same location as before for the counter injection. As can be seen in Fig. \& the solution to the equation of motion now takes on a



Figure 2: Quasi-periodic orbit of a counter-injected deuterium beam ion. The dashed line is the limiter. 


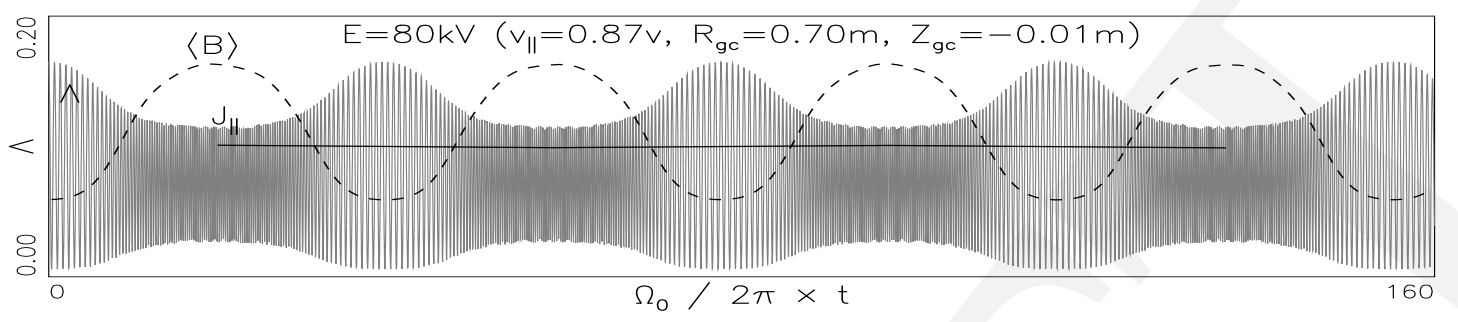

Figure 3: Adiabatic time evolution of normalized magnetic moment $\Lambda$ (thin, gray line), longitudinal invariant $J_{\|}$(solid line), and gyro-averaged magnetic field strength $\langle B\rangle$ (dashed line) for the orbit shown in Fig. 2 .

completely different character, as the adiabaticity condition is violated. The variation of the magnetic field along the orbit is actually less than it was for the counter-injected ion [see Fig. 5]; but because the gyro period is longer and the drift period is shorter, the change over one gyration becomes sufficiently large to destroy the adiabaticity of both $\Lambda$ and $J_{\|}$.



Figure 4: Chaotic orbit of a co-injected beam ion.
We now move the gyro center five centimeters outward, to the edge of the magnetic well. In the magnetic well the $B$ contours are nested, closed loops and roughly overlap the flux surfaces. The variation of $B$ along a flux surface is thus greatly reduced. Moving closer to the magnetic well, the adiabaticity parameter will gradually decrease, and launching the ion at $R_{g c}=0.75 \mathrm{~m}$, the character of the motion is again changed [see Fig. 6]. Notice how the ion shifts between three different quasi-periodic orbits. This behavior can be understood by inspecting the time evolution of $\Lambda$ and $J_{\|}$[see Fig. 7]. As can be seen, $\Lambda$ does not oscillate around a constant average and is thus non-adiabatic. Instead it jumps between three different gyro averages, explaining the peculiar ion orbit. In contrast, $J_{\|}$oscillates with a period of three drift periods around a constant average and thus qualifies as an adiabatic invariant. This phenomenon, with non-adiabatic $\Lambda$ but adiabatic $J_{\|}$ is known as super-adiabaticity [11. Note that in the super-adiabatic regime, the longitudinal invariant $J_{\|}$ replaces the magnetic moment as the third constant of motion, and the motion remains integrable. The confinement of super-adiabatic ions should thus be similar to that of adiabatic ions. As the adiabaticity parameter is further reduced and we leave the super-adiabatic regime, however, only two constants of motion will remain, and the confinement will be drastically decreased as the motion becomes chaotic. 


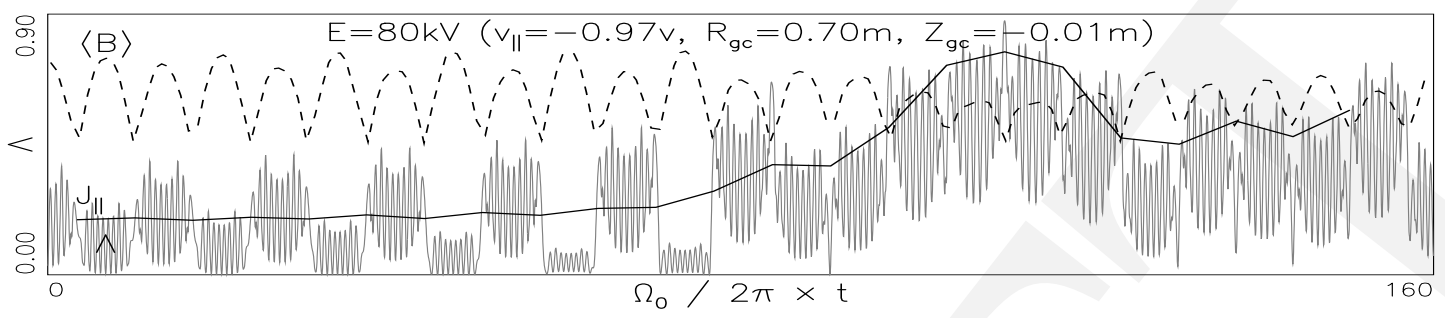

Figure 5: Non-adiabatic time evolution of $\Lambda$ and $J_{\|}$for the orbit shown in Fig. 田 $\Lambda$ exhibits characteristic, erratic jumps as $\langle B\rangle$ passes through local minima.

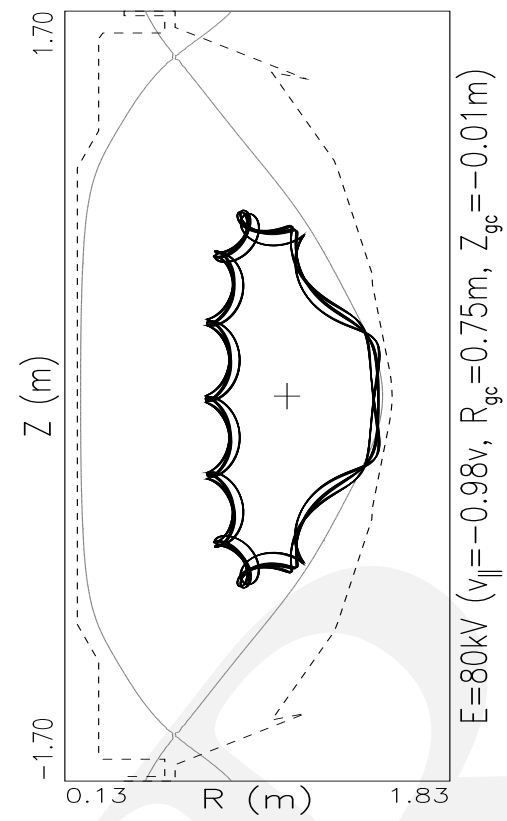

Figure 6: Almost quasi-periodic orbit of a superadiabatic co-injected beam ion on the edge of the magnetic well.

The non-adiabatic beam ion shown in Fig. 1 intersects the limiter, and it would have done so even if it had been adiabatic. This seems to be typical for NSTX: the non-adiabaticity only affects $80 \mathrm{kV}$ beam ions already following loss orbits and should thus not have any substantial, negative effect on the confinement. The majority of the confined beam ions are super-adiabatic, similar to the orbit in Fig. 6. In fact, even orbits deep inside the well have a slightly non-adiabatic magnetic moment.
In START, co-injection of a $30 \mathrm{kV}$ hydrogen beam was found to have $\sim 60 \%$ absorption efficiency [12]. Based on numerical simulations, these beam ions were claimed to be strongly non-adiabatic. This would seem inconsistent with the good confinement and we note that Fig. 3a of Ref. [12], which shows the time evolution of the magnetic moment for a typical beam ion and is said to demonstrate the non-adiabaticity, in fact indicates super-adiabatic, or possibly even fully adiabatic, beam ions.

If the beam energy is increased to $110 \mathrm{kV}$ in NSTX, the situation remains essentially the same: the vast majority of the beam ions that are not promptly lost, are super-adiabatic, and their motion is consequently bounded. However, in future, burning-plasma experiments, such as the VNS [10], the fusion-born $\alpha$-particles will display the whole range of dynamics: adiabatic, super-adiabatic, and non-adiabatic. As an example, we show the time evolution of the magnetic moment for an alpha, born just below the midplane and three centimeters outside of the magnetic axis, for a $38 \% \beta$ VNS equilibrium [see Fig. 8]. In addition to possible direct non-adiabatic losses when a giant jump in $\Lambda$ causes transition to a loss orbit, there is also an indirect loss mechanism. As can be seen, the jumps in $\Lambda$ force the $\alpha$ back and forth across the trapped-passing boundary. When driven by ion-ion collisions, this is of course the process that generates neo-classical transport. For fusion-born $\alpha$-particles, the drift period is many orders of magnitude shorter than the collisional timescale, and the non-adiabatic transport 


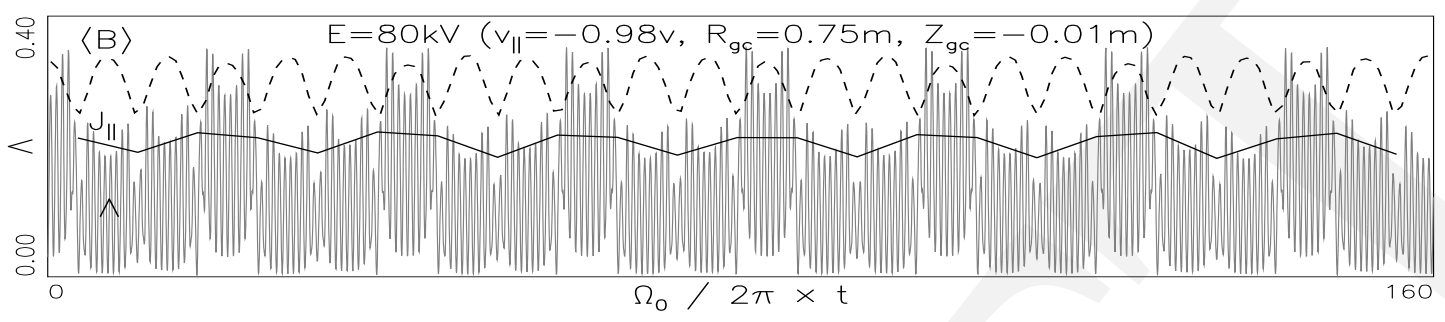

Figure 7: Non-adiabatic time evolution of $\Lambda$ but adiabatic time evolution of $J_{\|}$for the orbit shown in Fig. 6 .

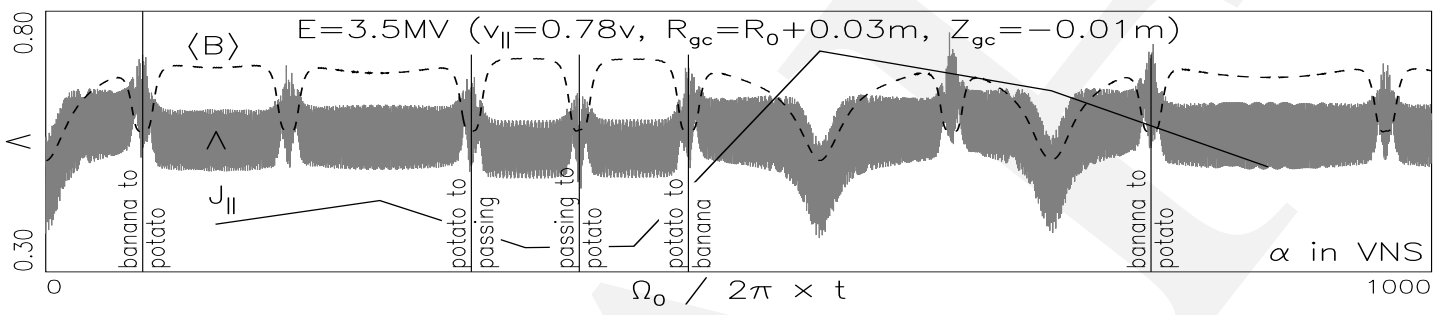

Figure 8: Non-adiabatic time evolution of $\Lambda$ and $J_{\|}$for fusion $\alpha$ in VNS.

mechanism described above can thus be expected to affect the overall $\alpha$ confinement. Yet another effect to consider is the Arnold diffusion [13], which might be important if the magnetic-field ripple is large enough to destroy the axi-symmetry.

Acknowledgment: This Letter has benefited greatly from the insightful comments of Fred Jaeger. The magnetic-equilibrium data was kindly provided by Dennis Strickler. This research was sponsored by the Oak Ridge National Laboratory, managed by UT-Battelle, LLC, for the U.S. Department of Energy under contract DE-AC05-00OR22725. Research was supported in part by an appointment to the ORNL Postdoctoral Research Associates Program, administered jointly by Oak Ridge National Laboratory and the Oak Ridge Institute for Science and Education. 


\section{References}

[1] Hannes Alfvén, "On the motion of a charged particle in a magnetic field", Ark. f. mat., astr. o. fysik 27 A (22), (1940) 1-20; Hannes Alfvén, "Cosmical electrodynamics", Oxford University Press, 1950.

[2] C. S. Gardner, "Adiabatic invariants of periodic classical systems", Phys. Rev. 115 (4), (1959) 791-794; T. G. Northrop, and E. Teller, "Stability of the adiabatic motion of charged particles in the Earth's field", Phys. Rev. 117 (1), (1960) $215-225$.

[3] A. Garren, R. J. Riddell, L. Smith, G. Bing, L. R. Henrich, T. G. Northrop, and J. E. Roberts, "Individual particle motion and the effect of scattering in an axially symmetric magnetic field", in Proceedings of the Second International Conference on Peaceful Uses of Atomic Energy, Vol. 31, (1958) pp. 65-71; R. J. Hastie, G. D. Hobbs, and J. B. Taylor, "Non-adiabatic behaviour of particles in inhomogeneous magnetic fields", in Proceedings of the Third International Conference on Plasma Physics and Controlled Nuclear Fusion, Vol. 1, IAEA, Vienna (1969) pp. 389-401.

[4] V. I. Arnold, "Small denominators and problems of stability of motion in classical and celestial mechanics", Russian Math. Surveys 18 (6), (1963) 85-191; J. Moser, "On invariant curves of area-preserving mappings of an annulus", Nachr. Akad. Wiss., Göttingen: Math. Phys. IIa (1), (1962) 1-20.

[5] B. V. Chirikov, "Resonance processes in magnetic traps", J. Nucl. Energy, Part C: Plasma Physics 1, (1960) 253-260; G. M. Zaslavskii, and B. V. Chirikov, "Stochastic instability of nonlinear oscillations", Sov. Phys. Usp. 14, (1972) 549-568; B. V. Chirikov, "Stability of motion of a charged particle in a magnetic confinement system", Sov. J. Plasma Phys. 4 (3), (1978) 289300; B. V. Chirikov, "A universal instability of many-dimensional oscillator systems", Phys. Reports 52 (5), (1979) 263-379.
[6] Y-K. M. Peng, and D. J. Strickler, "Features of spherical torus plasmas", Nucl. Fusion 26, (1986) 769-777; Y-K. M. Peng, "The physics of spherical torus plasmas", Phys. Plasmas 7, (2000) 1681-1692.

[7] A. Sykes, et al., "First results from the START experiment", Nucl. Fusion 32, (1992) 694-699; A. Sykes, et al., "The spherical tokamak programme at Culham", Nucl. Fusion 39, (1999) 1271-1281.

[8] A. Sykes, et al., "First physics results from the MAST mega-amp spherical tokamak", Phys. Plasmas 8, (2001) 2101-2106.

[9] S. M. Kaye, et al., "Physics design of the national spherical torus experiment", Fus. Tech. 36, (1999) 16-37; S. M. Kaye, et al., "Initial physics results from the national spherical torus experiment", Phys. Plasmas 8, (2001) 1977 1987.

[10] Y-K. M. Peng, "Spherical torus pathway to fusion power", J. Fusion Energy 17, (1998) 45-59; Y-K. M. Peng, "Science and technology of the 10 MA spherical tori", Nucl. Fusion 40, (2000) $583-587$.

[11] M. N. Rosenbluth, "Superadiabaticity in mirror machines", Phys. Rev. Lett. 29, (1972) 408-410.

[12] R. J. Akers, et al., "Neutral beam heating in the START spherical tokamak", Nucl. Fusion xx, (2001) $\mathrm{xxx}-\mathrm{xxx}$

[13] V. I. Arnold, "Instability of dynamical systems with several degrees of freedom", Sov. Math. 5, (1964) 581-585. 